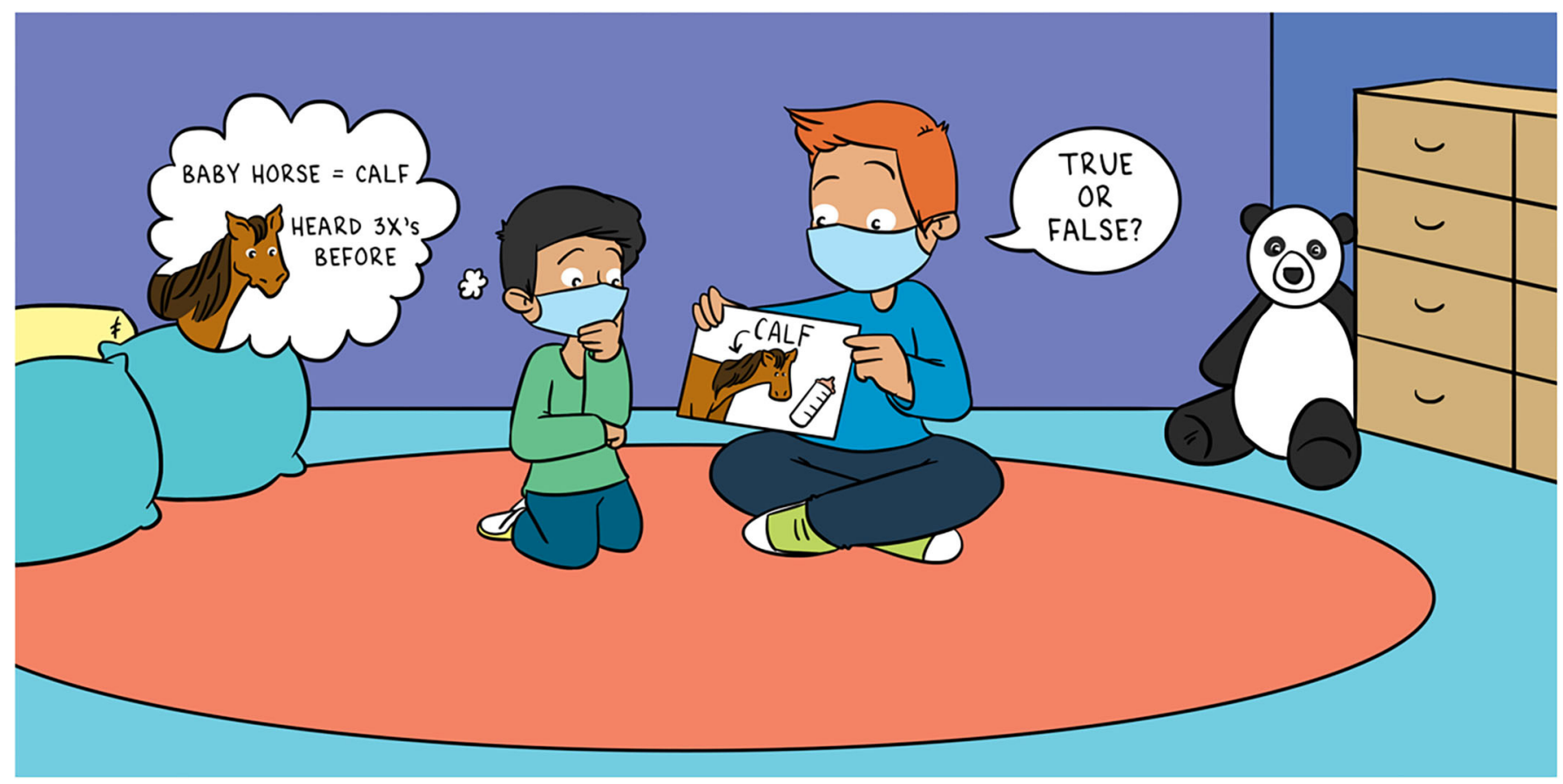

\title{
HOW REPETITION AFFECTS WHAT KIDS AND ADULTS BELIEVE
}

\section{Raunak M. Pillai ${ }^{*}$, Carrie L. Sherry and Lisa K. Fazio}

Department of Psychology and Human Development, Vanderbilt University, Nashville, TN, United States

\section{YOUNG REVIEWERS:}

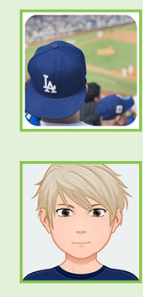

AGE: 9

RILEY

AGE: 8
How do you know that something is true? Is it because you learned it in school? Is it because you heard it before? Our brains can remember a lot of information, but we do not always use this information when deciding what is true. Sometimes we use shortcuts like, "Have I heard this before?" rather than thinking about what we know. These shortcuts can lead us to make mistakes-like thinking that a false statement is true just because you have heard it many times. Researchers call this the illusory truth effect. People are more likely to believe information they have heard multiple times. This happens even when people should know that what they are hearing is wrong! They already have the correct information stored in memory, but repetition makes them think that false information is true. In this article, we discuss why this happens, and how this might affect kids like you! 


\section{REPETITION MAKES PEOPLE BELIEVE SOMETHING IS TRUE}

What was the last thing you had to eat? That question probably was not too hard to answer, and that is because human brains have a powerful capacity for memory. Our brains take in information like sights and sounds, or ideas and concepts, and then store them until we need to think about them again. This is a really helpful ability, and it usually serves us well. Our memories help us when we are reading a book and want to think back to what happened in the previous chapter. They also help when we are playing a game and know all of the rules even though we learned them months or years ago. In fact, you are using your memory right now as you read this article, to store the main ideas in your brain and hopefully remember what you learn later!

In general, it is really helpful for people to have such strong memory systems. However, our memory system is not always perfect. Sometimes we forget things, like if we misplace something and cannot find it. Sometimes we think we know the right thing, but we really do not, like if we get a question wrong on a quiz when we were sure that we knew the right answer. And sometimes we fail to use our memories when we should. One example of failure to use our memories is something that researchers call the illusory truth effect. People who hear a false statement like "Leopards are the fastest land animal" multiple times are more likely to think that it is true than people who hear this statement only once [1]. Repeating something multiple times does not actually make it true. Your sister is not a monster no matter how many times you say she is! However, repetition can make statements feel like they are true.

Now, you might think this effect would only happen for facts or topics people do not know well or did not study in school. You might have even known right away that cheetahs, not leopards, are the fastest land animals. Unfortunately, the illusory truth effect happens even for information people should know is false, such as "The emu is an animal that is extinct" or "A microscope can help you get a good look at the stars." Hearing something false multiple times still makes people more likely to believe it, even if people's knowledge would tell them it is not true $[2,3]$ !

\section{DOES AGE MAKE A DIFFERENCE?}

Most of the research on the illusory truth effect has been done with adults. While it is important to study how adults think and how their memories work, it is also important to study kids' memories. By studying kids, we can get a better idea of how our brains change as we grow older. This is the main goal of scientists like us, who work in developmental psychology; we study how people's minds, well, develop! To find out how our brains develop in relation to the 
Table 1

Examples of true or false statements used in our experiment.

\begin{tabular}{lll} 
Difficulty & True & False \\
\hline Easy & A calf is a baby cow. & A calf is a baby horse. \\
Medium & Reptiles are cold-blooded animals & Birds are cold-blooded animals. \\
Difficult & $\begin{array}{l}\text { Cumulonimbus clouds often } \\
\text { bring thunderstorms. }\end{array}$ & $\begin{array}{l}\text { Cirrus clouds often bring } \\
\text { thunderstorms. }\end{array}$ \\
\hline
\end{tabular}

Table 1

illusory truth effect, we planned an experiment to help answer a few big questions: Do kids show the illusory truth effect? And if kids do show the effect, does it happen even for very young children? Or, do we need to be able to think about our own thinking (something only older children can do) in order to make the connection between repetition and truth?

\section{HOW DID WE TEST THE ILLUSORY TRUTH EFFECT?}

To figure out whether kids also show the illusory truth effect, we first had to pick the ages we were interested in studying. In this experiment, we chose 5-year-old kids, 10-year-old kids, and college-aged adults, to compare groups that think very differently. We used 5-year-olds as our youngest age group because children younger than 5 did not really understand how our experiment worked!

Next, we needed some statements to test what people believed. We focused on simple statements about nature, with different levels of difficulty. Some of these statements were ones that even the 5 -year-olds would probably know were true or false (easy). Some would probably be known by both 10-year-olds and adults (medium). And others even the adults would not know if they were true or false (difficult). We used 48 statements in all. Look at Table 1 for examples of some of the true and false statements we used.

Finally, we needed some way to see whether people thought the statements were true or not. That is the easiest part-we just asked people what they thought! In this experiment, people were asked if each statement was "true" or "not true" and if they were "very sure" or "not so sure" of the answer they chose.

Once we had all the pieces - the people being tested, the statements to test them on, and the way to test them-we put them all together to create our experiment! First, we got the adults and kids to come into our laboratory and agree to take part in the study. Then, we had the participants listen to 24 of the 48 statements (12 were true and 12 were false). While they were listening, everybody chose whether they thought each statement was "interesting" or "not interesting" (just so they could have something to do while listening). The statements were presented through headphones by "Ruby the Robot." Before listening, everyone was told, "Ruby knows a lot about some things, and not a lot 
Figure 1

In our experiment,

Ruby the Robot recited true or false statements to participants, who then had to rate the statements as "true" or "not true" and choose whether they were "very sure" or "not so sure" of their answers.

\section{Figure 2}

Percentages of new and repeated statements rated "true" for each age group. Repeated statements (in orange) were more likely to be rated "true" than new statements (in blue), for all ages of participants. This means that the illusory truth effect occurs for kids as young as 5 , as well as adults.

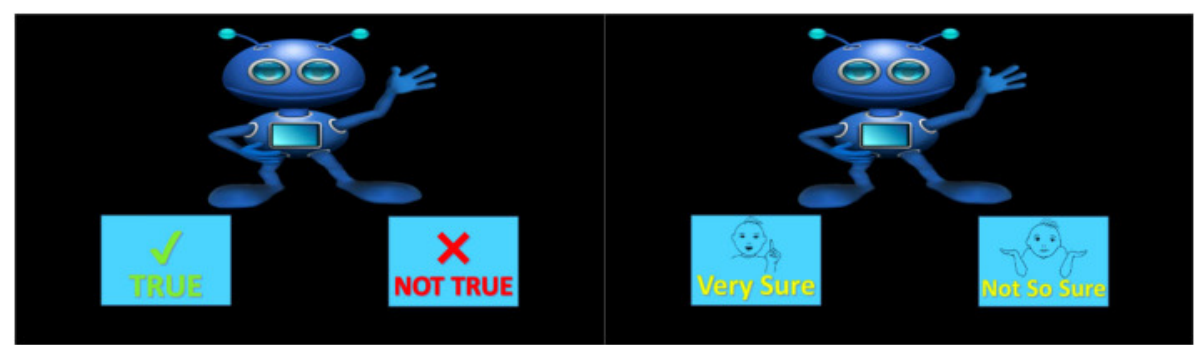

Figure 1

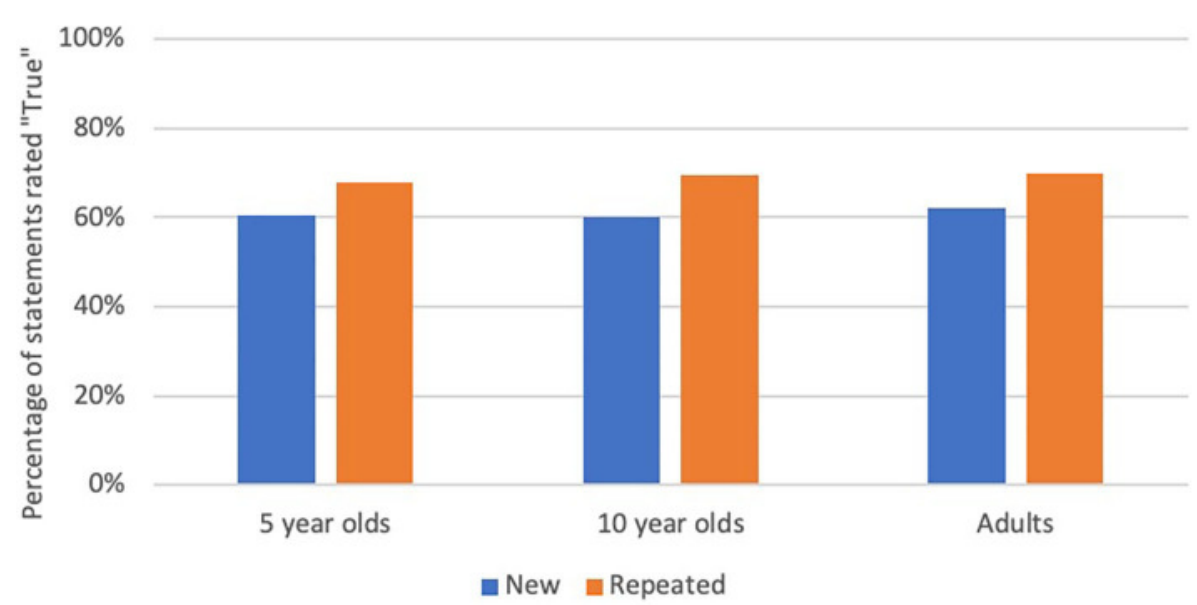

Figure 2

about other things. So, some of the things Ruby tells you will be true, and some will not be true."

After listening, the participants completed a maze or some puzzles to get their minds off the experiment for about a minute. Finally, they listened to Ruby recite all 48 statements-both the ones they had heard before and some new ones they were hearing for the first time. For each statement, we had them choose whether the statement was "true" or "not true," and then if they were "very sure" or "not so sure" about their answers (Figure 1).

\section{BOTH KIDS AND ADULTS EXPERIENCE THE ILLUSORY TRUTH EFFECT}

Our main finding was that no matter what age they were, the people we tested rated the repeated statements as "true" more often than statements that were new (Figure 2). In other words, hearing a statement more than once caused people of all ages to believe it more! This happened for both true and false statements. We expected, based on prior research, that the illusory truth effect would happen for adults, but the fact that it also happened for 5-year-old and 10-year-old kids was a new finding! 


\section{REPLICATION}

In scientific research, the repetition of an experiment to confirm findings or to ensure accuracy.
The other interesting finding was that everybody believed the repeated statements more, regardless of the difficulty level of the statements. In other words, repetition affected what the 5-year-old kids thought about the hard statements, what the 10-year-old kids thought about the medium statements, and even what the adults thought about the easy statements. Repetition even made the adults in our study more likely to believe simple statements like "A wasp is the insect that makes honey." This happened even though many preschoolers know that the real answer is bees!

\section{SO, WHAT DOES OUR RESEARCH MEAN?}

The main conclusion from our study is that the link between repetition and truth starts early on in life-at least by the time we are 5 years old. Five-year-olds who heard a statement twice were more likely to think that it was true, as were 10-year-olds and adults. We also confirmed that people do believe repeated facts, even when they should already know they are false. Since other studies had already found this to be true, this is called replication, and it means that the results of one experiment were found again by a different study. Because different experiments can sometimes lead to different results, replicated findings help provide additional evidence.

Overall, when developmental psychologists do this kind of memory research, it can help us understand how children learn and what factors influence what they think is true and false. So many things in our lives are repeated-from ads on TV to fun facts our friends may tell us. Some of these things are true, and some of them are false, so it is important for us to understand the way that this repetition affects how we remember the information!

\section{ORIGINAL SOURCE ARTICLE}

Fazio, L. K., and Sherry, C. L. 2020. The effect of repetition on truth judgments across development. Psychol. Sci. 31:1150-60. doi: 10.1177/0956797620939534

\section{REFERENCES}

1. Dechêne, A., Stahl, C., Hansen, J., and Wänke, M. 2010. The truth about the truth: a meta-analytic review of the truth effect. Pers. Soc. Psychol. Rev. 14:238-57. doi: 10.1177/1088868309352251

2. Fazio, L. K., Brashier, N. M., Payne, B. K., and Marsh, E. J. 2015. Knowledge does not protect against illusory truth. J. Exp. Psychol. Gen. 144:993-1002. doi: $10.1037 / x g e 0000098$ 
3. Fazio, L. K., Rand, D. G., and Pennycook, G. 2019. Repetition increases perceived truth equally for plausible and implausible statements. Psychon. Bull. Rev.

26:1705-10. doi: 10.3758/s13423-019-01651-4

SUBMITTED: 10 July 2020; ACCEPTED: 09 March 2021;

PUBLISHED ONLINE: 09 April 2021.

EDITED BY: Xi-Nian Zuo, Beijing Normal University, China

CITATION: Pillai RM, Sherry CL and Fazio LK (2021) How Repetition Affects What Kids and Adults Believe. Front. Young Minds 9:582203. doi: 10.3389/frym.2021. 582203

CONFLICT OF INTEREST: The authors declare that the research was conducted in the absence of any commercial or financial relationships that could be construed as a potential conflict of interest.

COPYRIGHT @ 2021 Pillai, Sherry and Fazio. This is an open-access article distributed under the terms of the Creative Commons Attribution License (CC BY). The use, distribution or reproduction in other forums is permitted, provided the original author(s) and the copyright owner(s) are credited and that the original publication in this journal is cited, in accordance with accepted academic practice. No use, distribution or reproduction is permitted which does not comply with these terms.

\section{YOUNG REVIEWERS}
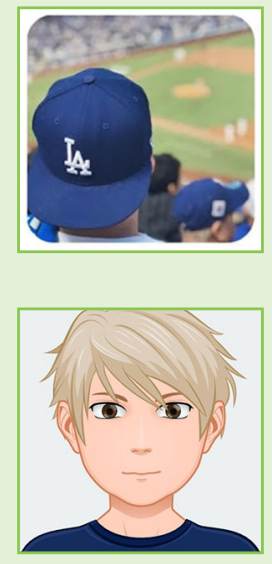

\section{AUSTIN, AGE: 9}

I am a young boy living in southern California. My favorite subject in school is Math and PE. On my free time I like to go outside, play sports, skateboard, and rollerblade. I joined Frontiers for Young Minds because I wanted to learn more about the brain and other parts of the human body.

\section{RILEY, AGE: 8}

Riley has a curious mind and loves learning about how things work. His favorite school subjects are math and science and he loves playing hockey, soccer, and golf. Riley enjoys reading both fiction and non-fiction books and sharing the facts and knowledge he learns. He wants to be a scientist when he grew up so he can make discoveries of his own.

\section{AUTHORS}

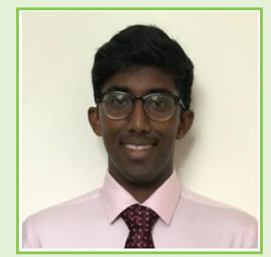

\section{RAUNAK M. PILLAI}

Raunak Pillai is a graduate student in psychology at Vanderbilt University. He works in the Building Knowledge Lab, studying how people learn from the kinds of information found in real-world contexts like the classroom. He is particularly interested in how people remember whether information they have encountered before is correct or incorrect. *raunak.m.pillai@vanderbilt.edu 


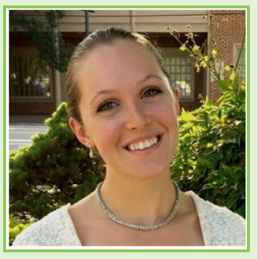

\section{CARRIE L. SHERRY}

Carrie Lorraine Sherry was the lab manager for the Building Knowledge Lab at the time this experiment was conducted. After taking some time to explore the processes of learning and memory under Dr. Jennifer Coffman at UNC and under Dr. Fazio at Vanderbilt, she is hoping to pursue further education in Cognitive Neuroscience when she moves to Houston with her husband. As a former teacher, she is especially interested in the thinking processes involved in inquiry-oriented learning.

\section{LISA K. FAZIO}

Dr. Lisa Fazio is an assistant professor of psychology at Vanderbilt University. Her Building Knowledge Lab studies how people learn new information, both true and false, and how to correct errors in people's knowledge. This includes how to reduce the negative effects of reading false information and how to increase classroom learning. Her research helps to support basic theories about learning and memory, while also having clear applications for journalists and teachers. 